\title{
Tips for constructing an effective title
}

\author{
Deliberate practice will, however, get you further.
}

( onstraints can lead to better outcomes. The freedom offered by a blank page may feel daunting, but the length restrictions and interpretability requirements of a single short sentence can make for a formidable challenge. You should welcome it. The job of a title for a scientific paper is not simply presentational; a well-crafted title expands the reach of the work, particularly to audiences who may benefit most from it.

Useful constraints for writing great titles come in different forms: scientific, linguistic and stylistic (the latter are usually imposed by the journal or publication venue, and by the medium of communication). Stylistic restrictions - in particular, the maximum number of characters, whether punctuation and non-standard abbreviations can be used, and whether declarative, imperative and interrogative sentences (and even dependent clauses) are allowed - are easiest to meet when considered first. No new acronyms, no colons, no -ing-ending verbs, British spelling and 130 characters at most? Keep calm and carry on.

Linguistic constraints typically have a common purpose: to save the reader effort. Shorter titles are not better if they are harder to read, convey too little, or miss an essential keyword. Refrain from biasing the reader by avoiding terms implying novelty or excitement - such as 'for the first time', 'dramatic', 'intriguing' and 'successful'. Think of specific wording rather than defaulting to general or ambiguous terms (such as 'system,' 'platform', 'engineering' and 'approach'). Pay attention to the most common meaning of each term in the context of the sentence; for example, 'by' and 'via' cannot always be used interchangeably: 'by' often identifies the 'how', and can suggest cause and effect'; 'via' (and 'through' and 'by means of') may not imply causality and may indicate that what comes afterwards is one option or a step among many. Make the title flow by avoiding adjective stacking, by using hyphenation to remove ambiguity, and by substituting jargon with common words when possible.

Scientific requisites can be more easily manoeuvred. As long as the title is accurate, there is substantial leeway as to how much needs to be conveyed. A general title is easier to construct, yet may suggest that the report is a review of previous work rather than conveying new findings, or it may fall flat by failing to communicate what is special or new in the work. Lifting up an apparently 'boring' \section{YOUR THESIS TITLE CONDENSING OVER HALF A DECADE OF}

WW.phdcomics.com JORGE CHMM (C) 2006

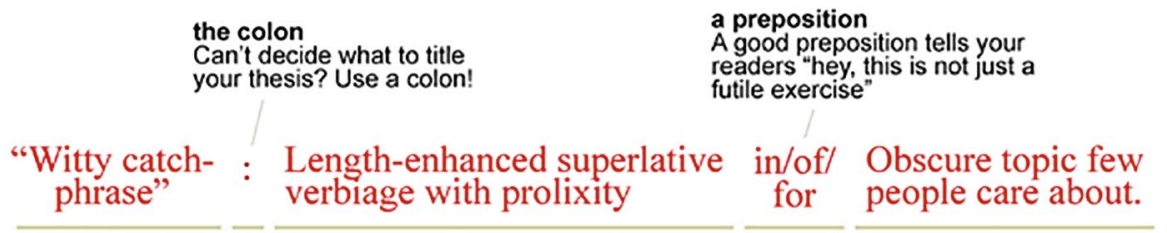

witty catchphrase

Makes people think you're

hip and culturally relevant.

Only marginally related to the

actual thesis? No problem.

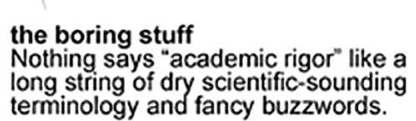

Nothing says "academic rigor" like a
long string of dry scientific-sounding terminology and fancy buzzwords.

\begin{abstract}
obscure topic
few people care

about

Sad, but true.
\end{abstract}

Credit: "Piled Higher and Deeper" by Jorge Cham www.phdcomics.com

title beyond what the evidence provides will not do the reader any favours; hence, refrain from claiming attributes or implications with terms such as 'with broad applicability', 'with clinical potential' and 'for point-of-care use' if the work only explored one application, the evidence is purely preclinical, and real-world settings haven't been considered. Also, regardless of whether the title is descriptive of the work or declares the main findings, avoid communicating bold assertiveness, unless the evidence strongly supports it. And being too specific or wordy (by using uncommon gene names or biological pathways for example) may alienate readers who may have gained from delving into the paper.

Constraints are mostly about 'what not to do' (pictured). So, what should one do to construct a great title? What are the hallmarks of it? Aim for scientific accuracy, interpretability and proper emphasis. And apply these three qualities with the intended audience in mind. An accurate and accessible title for an expert immunologist may be information-dense and use consensus nomenclature for $\mathrm{T}$ cell phenotypes; but the physics student interested in biology will disagree on the title's interpretability, regardless of how accurate it is. Think of the scientific knowledge of the average reader in the intended audience, and what the title needs to convey in order for them to decide whether to engage with the paper. For instance, for preclinical animal work, does the title need to state the animal model or the species? (Such as 'in mice' or 'in macaques.) And in diagnostics or therapeutics, does the title need to state the disease, sample type, or whether the biological material or species is 'human' or 'humanized'?

Proper emphasis is an underrated yet crucial quality. Is the work mainly about showing better performance, describing a new method, validating a technology, providing mechanistic understanding, or reporting a new application or functionality? A great title implicitly conveys the most suitable emphasis of the work; don't force the reader to read the abstract to figure it out.

Where to start? How does one go about condensing a great deal of new knowledge into a sentence that is accurate, accessible and has a suitable emphasis? Here are two tips: list the most important keywords those that make the work unique - and rank them by importance. Then, try to place the top-ranked terms at the beginning and at the end of the title. A caveat: these tips don't always work.

What really works is deliberate practice. Write titles with purpose, repeatedly; and seek useful feedback. And it doesn't hurt to examine edited scientific titles. We can offer some examples: search for published Articles with a peer-review file included as Supplementary information (the file provides the reviewer reports, author rebuttal letters and, in the case of Nature Biomedical Engineering, the editorial decision letters), and compare the original and published versions of the Articles' titles. Are we living up to what we preach?

Published online: 21 February 2022 https://doi.org/10.1038/s41551-022-00858-6 